I UC STUDI ES

ISSN 1813-7733

Vol. - 4, December 2007

Published in April 2008 (p 71-86)

\title{
Display Unit for Bangla Characters
}

\author{
Mohammad Osiur Rahman* \\ Mohammad Aktaruzzaman Khan ${ }^{* *}$
}

\begin{abstract}
Segmented representation is used in lieu of the dot matrices representation for alphanumeric characters of different languages. Due to concerns of cost, power loss and design complexity, the segmented representation becomes more popular over the dot matrices. There are very limited display units for English, Arabic, and Bangla alphanumeric characters. 18-segments display is normally used to display the entire alphabetic character of English. But there is no standard display unit for Bangla Alphabetic Characters. Although recently different segmented display models for Bangla characters have been proposed, the quality of the characters stay below the mark. In this paper, 26-segments display for Bangla alphabetic character has been proposed. From the review it appears that it is the first proposed display in the world for Bangla Alphabetic Character. Here a grid structure consisting of 26 segments has been discovered. All Bangla characters vowel and consonants can be characterized by using this grid structure. As there can be at least vowels (12), consonants (39), punctuation, and some other symbols to be displayed, 6-bit inputs are used to represent each character. After analyzing which segments will be activated for which character, appropriate logic function and circuits have been derived in order to display each Bangla character. Finally, a comparative analysis is made among the different proposed segmented display models to find out the cost and effectiveness in terms of display quality, power loss, and design complexity.
\end{abstract}

Keywords: 7-segment, 12-segment, 17-segment, 18segment, non-overlapping segments, K-map.

\section{Introduction}

Now-a-days Bangla Alphabetic Characters are represented using dotmatrics system $[1,2]$ where a large number of dots are to be

* Assistant Professor, Dept. of Computer Science \& Engineering, University of Chittagong and Ex- Assistant Professor, Dept. of Computer Science \& Engineering, IIUC

** Lecturer, Department of Business Administration, IIUC. 
manipulated. On the other hand for English Alphanumeric LED displays are available in three common formats [1]. For displaying only numbers and hexadecimal letters, simple 7-segment displays such as that shown in [1] are used. To display numbers and the entire alphabetic, 18-segments displays such as that shown in [1] or $5 \times 7$ dotmatrix displays such as that shown in [1] can be used. 11-segment display for Bangla and English numerals [3,4], 12-segment for Bangla, English, and Arabic numerals, 9-segment for only English and Arabic digits [3], 9-segment [5] and 10-segment [6,7,8] for Bangla digits and 16-segments for multilingual [9] are already proposed. Since, Bangla Alphabetic characters are represented using dot metrics as a result the cost of the display unit increases due to storage space, a large number of dots, power loss, and design complexity. But recently very few researchers address the point to represent Bangla Alphabetic characters using segmented display unit $[10,11,12,13]$. Still there is no standard segmented display unit for Bangla Alphabetic characters. Moreover, to present the proposed 26-segmented display system is the most perfect and effective among all other display models $[10,11,12,13]$ in terms of display quality, power loss, and design complexity. In this proposed 26-segment display system, we have to manipulate only 26 segments, which saves considerable amount of money or storage space [14]. In this display unit segments don't intersect each other. It is easy to implement for any display unit.

\section{Previous Proposed Segment Display Models For Bangla Characters}

A.K.M. Najmul Islam et. al. proposed 17-segment display unit [10] for Bangla vowels. In Figure 1(a) shows the 17-segment display unit and Figure 1(b) shows its character sets. From its character sets it is observed that vowel $F$ is not clear enough. The quality of appearance is not good enough for all characters except $A, A \vee D$, and $E$ characters. Drawbacks of 17-segment for Bangla vowels are that the characters are not clear enough and the quality of characters is not good enough.

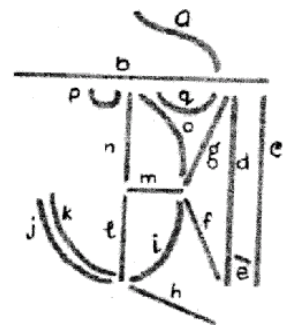

(a) 


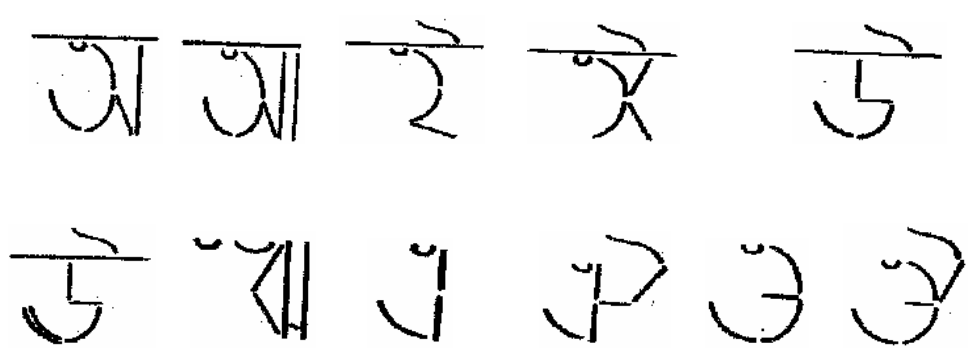

(b)

Figure 1: (a) 17-segment display for Bangla Vowels (b) Character Sets for 17segment display for Bangla Vowels

Salauddin Mohammad Masum et. al. proposed a 14-Segment display model [11] only for vowels. In Figure 2(a) shows the 14-segment display unit and Figure 12(b) shows its character sets. It covers only 11 Bangla vowels. The model is mainly based on straight lines. But the traditional Bangla vowel alphabets are not based only on straight lines. Many of them are based rather curved lines to a great extend.

So, 14-segment display system for Bangla vowels cannot be accepted a standard in the display system because, here, the proposed vowels are asymmetrical, and dissimilar and are, rather, not familiar to the users. The proposed alphabets are, also, not identical to corresponding Bangla ones.

This 14-segment model is not full, rather partial, as it is lacking in Bangla consonants and digits.

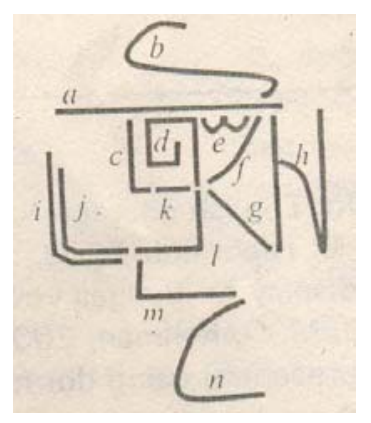

(a) 


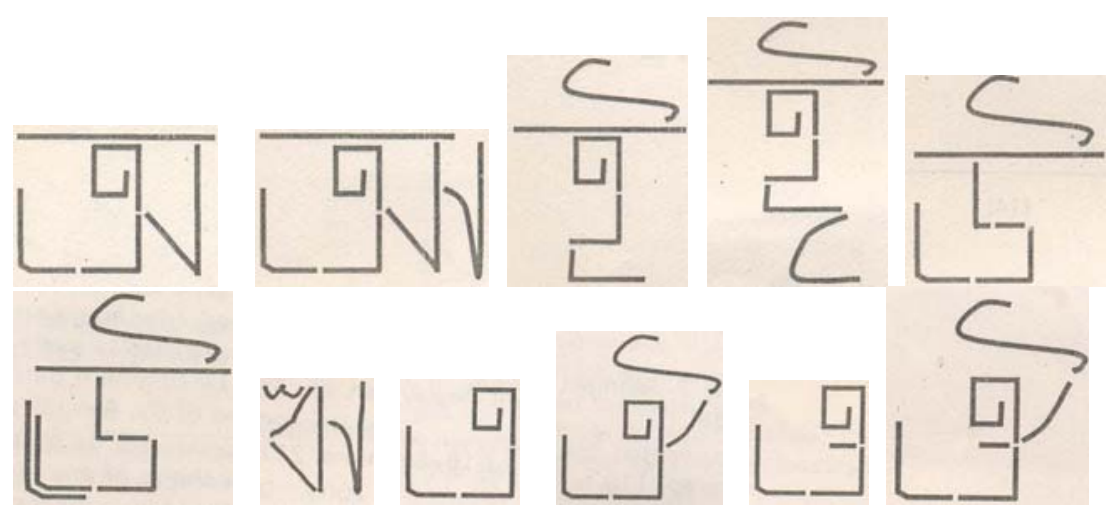

(b)

Figure 2: (a) 14-segment display for Bangla Vowels (b) Character Sets for 14-segment display for Bangla Vowels

Samiran Mahmud et. al. proposed 31-segment display model [12] for Bangla characters. In Figure 3(a) shows the 31-segment display unit and Figure 3(b) shows its character sets. The proposers claim that this display system has been proposed for Bangla characters, both digits and alphabets. But, here, we find 28 consonants and 6 digits only. Hence, it lacks 11 consonants and 4 digits. So, it does not cover all the Bangla alphabets and digits.

Many of the proposed characters are dissimilar to the original Bangla characters, such as $P, C, f, T, U$, and $V$. These are not also familiar to the users.

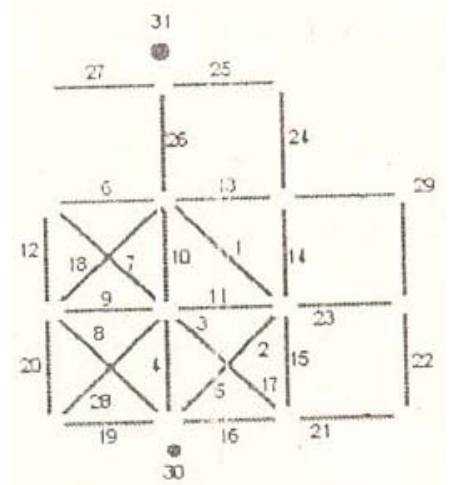

(a) 

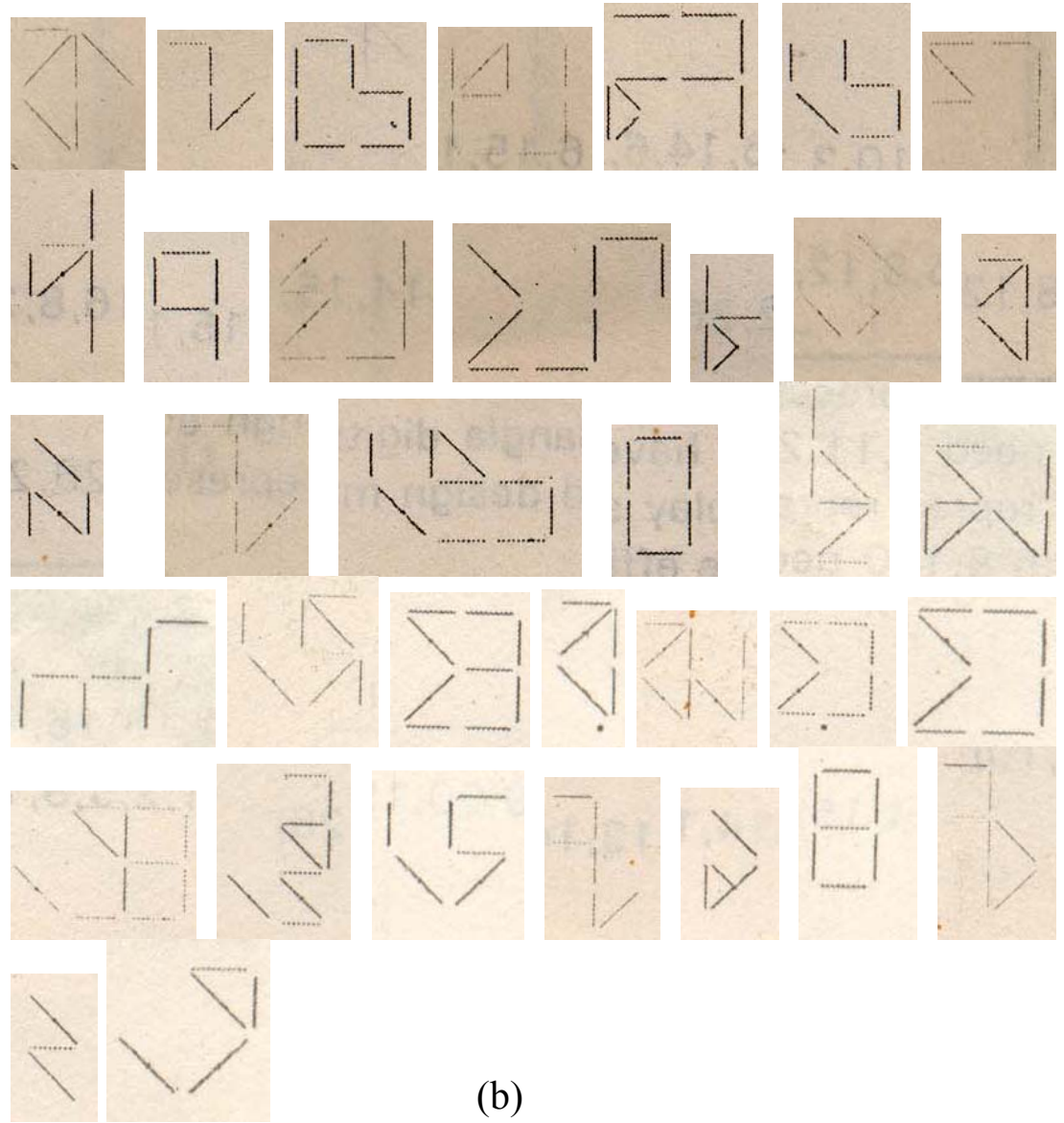

(b)

Figure 3: (a) 31-segment display for Bangla Characters (b) Character Sets for 31-segment display for Bangla Characters

Salauddin Mohammad Masum et. al. proposed 44-segment display model [13] for consonants. In Figure 4(a) shows the 44-segment display unit and Figure 4(b) shows its character sets. This model is proposed only for consonants. Some of the characters of this model are better than those of 31-segment display model. But some other characters are based on straight lines and, hence, are dissimilar to the original Bangla consonants such as $K, Q, I, k, n, Z, V, g, f, r$, and $u$ 
IIUC Studies, Vol. 4

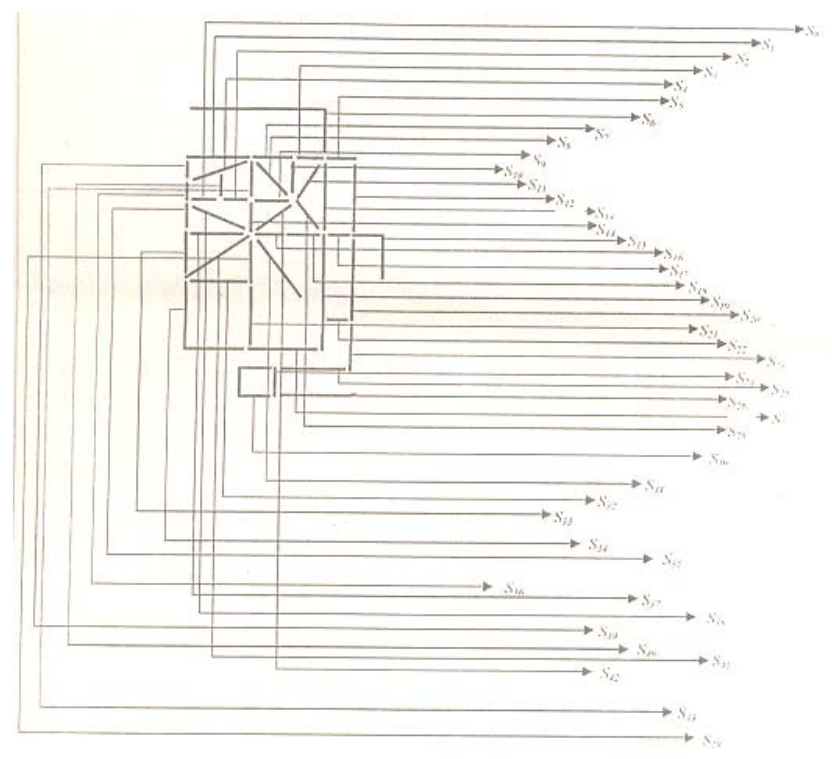

(a)

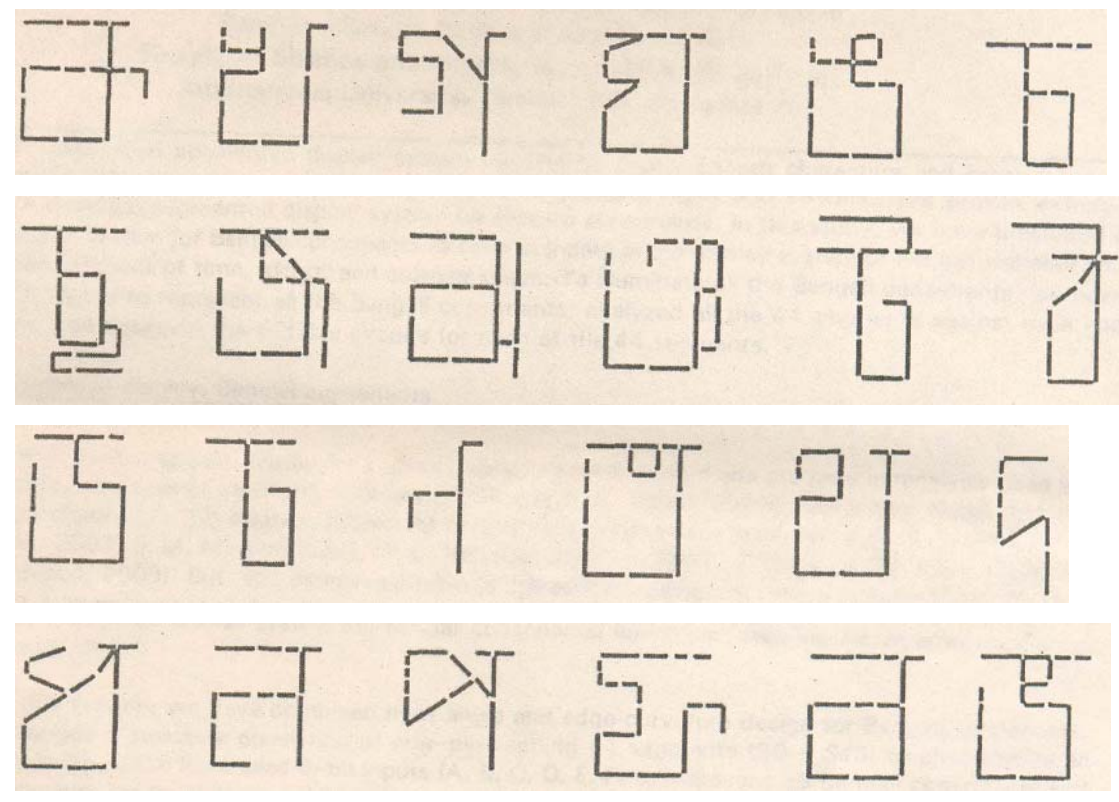




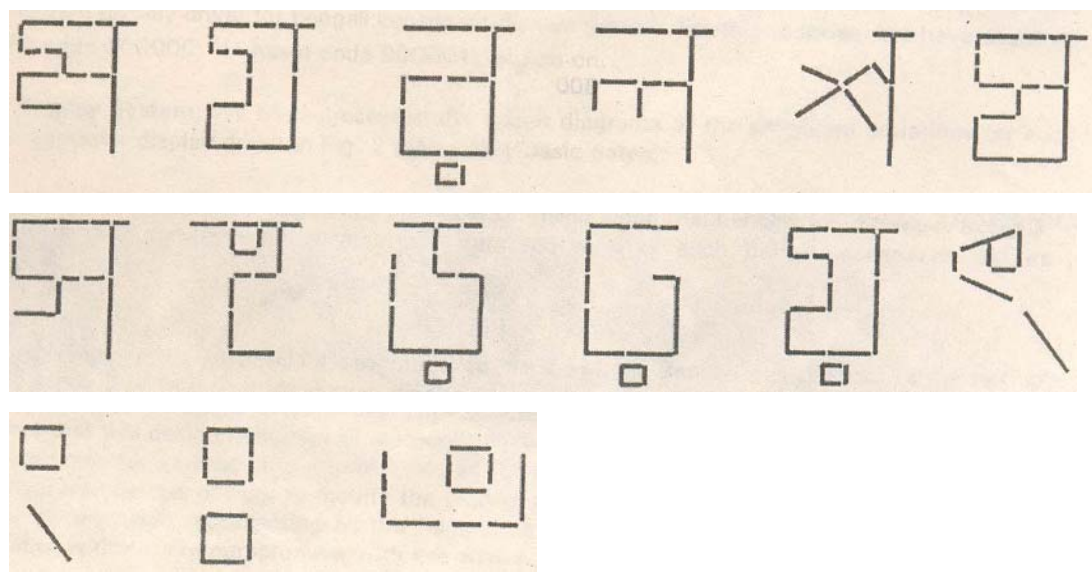

(b)

Figure 4: (a) 44-segment display only for Bangla consonants (b) Character Sets for 44-segment display only for Bangla consonants

\section{26-segment Display}

We have discovered a grid structure consisting of 26 segments (shown in Figure 5). All Bangla characters vowel and consonants can be characterized by using this grid structure. As there can be at least vowels (12) and consonants (39) and some special symbols to be displayed, 6-bits input are used to represent each character. After analyzing which segments will be activated for which character, appropriate logic function and circuits have been derived in order to display each Bangla character.

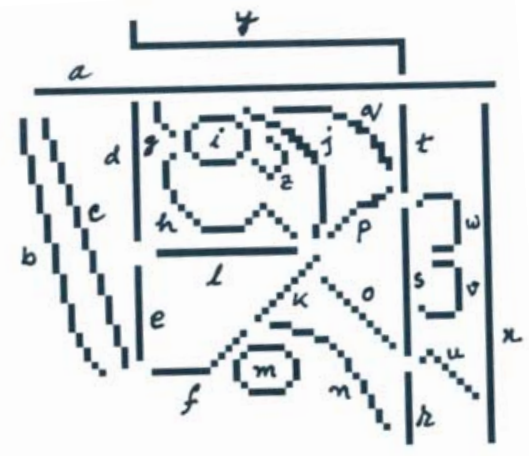


Figure 5: The Model of 26-segment for Bangla Alphabetic Characters Display

\section{Characterization for Bangla Characters By using Segments}

In Table 4.1 we represent all the Bangla alphabetic characters by using our proposed 26-segment display. From the table we summarized Table 4.2 that represents the active segments of corresponding Bangla alphabetic characters.

By using 7-segment display all the English digits (0-9) can be displayed and by using 18-segment Alphanumeric Character of English can be displayed. But there is no display system for Bangla. In our proposed display system, all Bangla Alphabetic characters can be represented by using some or all the 26-segments a, b, c, d, e, f, g, h, i, $\mathrm{j}, \mathrm{k}, \mathrm{l}, \mathrm{m}, \mathrm{n}, \mathrm{o}, \mathrm{p}, \mathrm{q}, \mathrm{r}, \mathrm{s}, \mathrm{t}, \mathrm{u}, \mathrm{v}, \mathrm{w}, \mathrm{x}, \mathrm{y}, \mathrm{z}$ which are non-overlapping. The structure of non-overlapping segments is shown in figure 5.

To represent Bangla alphabetic characters the following segments are activated for respective digits from our proposed 26-segment display:

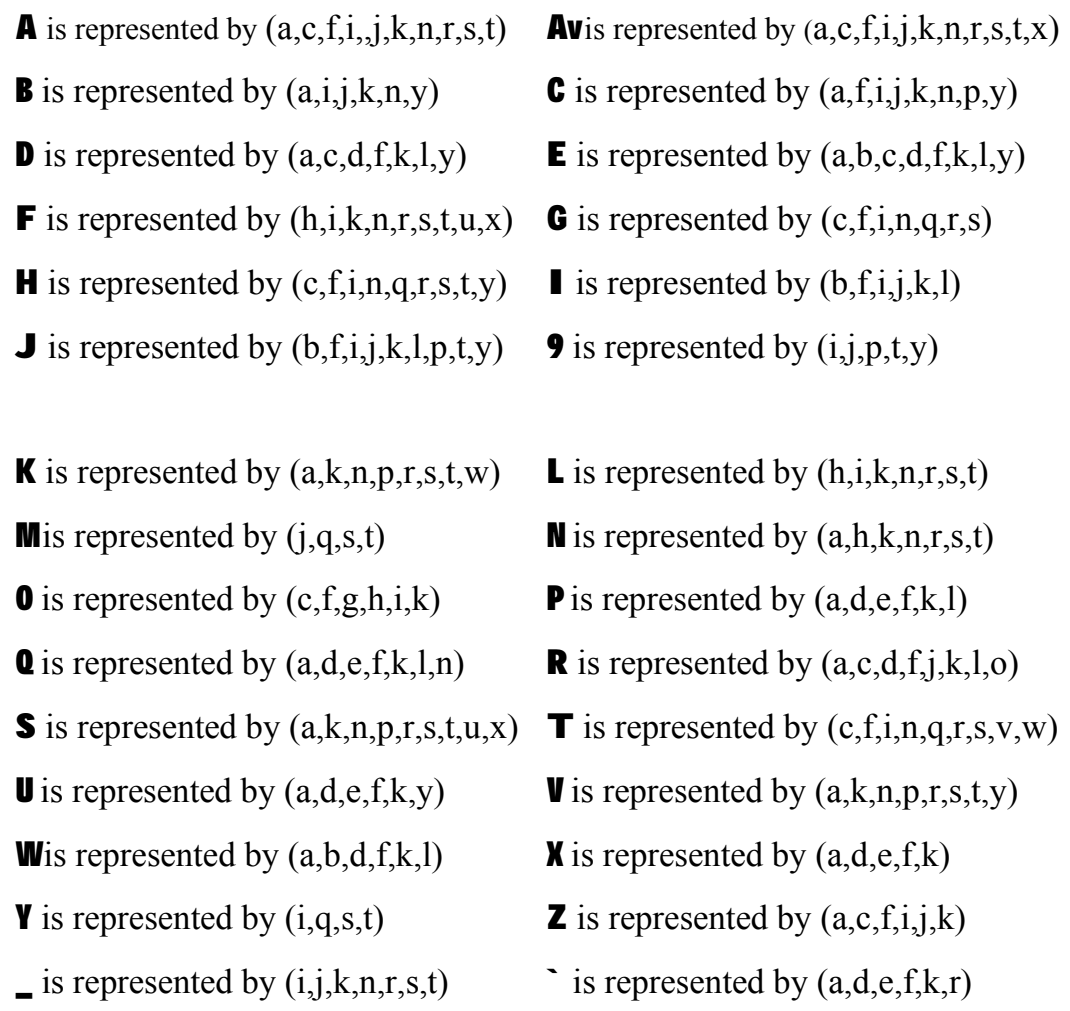




$\begin{array}{ll}a \text { is represented by }(\mathrm{h}, \mathrm{i}, \mathrm{k}, \mathrm{n}, \mathrm{p}, \mathrm{r}, \mathrm{s}, \mathrm{t}) & \mathrm{b} \text { is represented by }(\mathrm{a}, \mathrm{m}, \mathrm{n}, \mathrm{r}, \mathrm{s}, \mathrm{t}) \\ \mathrm{c} \text { is represented by }(\mathrm{j}, \mathrm{p}, \mathrm{q}, \mathrm{s}, \mathrm{t}) & d \text { is represented by }(\mathrm{a}, \mathrm{j}, \mathrm{k}, \mathrm{n}, \mathrm{r}, \mathrm{s}, \mathrm{v}) \\ \mathrm{e} \text { is represented by }(\mathrm{a}, \mathrm{k}, \mathrm{n}, \mathrm{p}, \mathrm{r}, \mathrm{s}, \mathrm{t}) & f \text { is represented by }(\mathrm{a}, \mathrm{b}, \mathrm{f}, \mathrm{h}, \mathrm{i}, \mathrm{k}) \\ \mathrm{g} \text { is represented by }(\mathrm{a}, \mathrm{h}, \mathrm{k}, \mathrm{m}, \mathrm{n}, \mathrm{r}, \mathrm{s}, \mathrm{t}) & \mathrm{h} \text { is represented by }(\mathrm{a}, \mathrm{j}, \mathrm{k}, \mathrm{n}, \mathrm{r}, \mathrm{s}, \mathrm{t}) \\ \mathrm{i} \text { is represented by }(\mathrm{a}, \mathrm{k}, \mathrm{m}, \mathrm{n}, \mathrm{p}, \mathrm{r}, \mathrm{s}, \mathrm{t}) & \mathrm{j} \text { is represented by }(\mathrm{a}, \mathrm{m}, \mathrm{n}, \mathrm{r}, \mathrm{u}, \mathrm{x}) \\ \mathrm{k} \text { is represented by }(\mathrm{g}, \mathrm{i}, \mathrm{q}, \mathrm{s}, \mathrm{t}, \mathrm{z}) & \mathrm{I} \text { is represented by }(\mathrm{a}, \mathrm{j}, \mathrm{k}, \mathrm{n}, \mathrm{o}, \mathrm{r}, \mathrm{s}, \mathrm{t}) \\ \mathrm{m} \text { is represented by }(\mathrm{a}, \mathrm{f}, \mathrm{j}, \mathrm{k}, \mathrm{n}, \mathrm{r}, \mathrm{s}, \mathrm{t}) & \mathrm{n} \text { is represented by }(\mathrm{a}, \mathrm{i}, \mathrm{j}, \mathrm{k}, \mathrm{n}) \\ \mathrm{o} \text { is represented by }(\mathrm{a}, \mathrm{b}, \mathrm{d}, \mathrm{f}, \mathrm{k}, \mathrm{l}, \mathrm{m}) & \mathrm{p} \text { is represented by }(\mathrm{a}, \mathrm{d}, \mathrm{e}, \mathrm{f}, \mathrm{k}, \mathrm{m}) \\ \mathrm{q} \text { is represented by }(\mathrm{a}, \mathrm{j}, \mathrm{k}, \mathrm{m}, \mathrm{n}, \mathrm{r}, \mathrm{s}, \mathrm{t}) & \mathrm{r} \text { is represented by }(\mathrm{f}, \mathrm{h}, \mathrm{i}, \mathrm{k}) \\ \mathrm{s} \text { is represented by }(\mathrm{c}, \mathrm{i}) & \mathrm{t} \text { is represented by }(\mathrm{i}, \mathrm{m}) \\ \mathrm{U} \text { is represented by }(\mathrm{c}, \mathrm{f}, \mathrm{i}, \mathrm{k}, \mathrm{p}) & \end{array}$


Table 4.1: Representation of Bangla Alphabetic Characters by using our proposed 26-segment display.

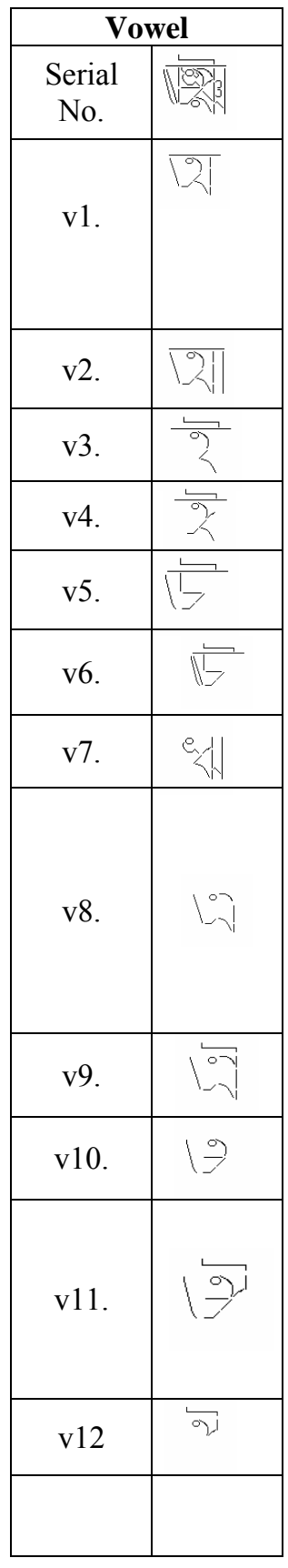

\begin{tabular}{|c|c|c|c|c|c|}
\hline \multicolumn{6}{|c|}{ Consonant } \\
\hline $\begin{array}{l}\text { Serial } \\
\text { No. }\end{array}$ & $\sqrt{\sqrt{207}}$ & $\begin{array}{c}\text { Serial } \\
\text { No. }\end{array}$ & 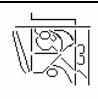 & $\begin{array}{c}\text { Serial } \\
\text { No. }\end{array}$ & $\sqrt{\sqrt{20 \pi}}$ \\
\hline $\mathrm{c} 1$ & & c14 & or & c27 & 61 \\
\hline c2 & $\sum_{j} \mid$ & c15 & oy & c28 & $\mid \partial_{1}$ \\
\hline c3 & $y$ & c16 & 9 & c29 & |y \\
\hline c4 & 41 & c17 & $2 \mid$ & c30 & $z_{1}$ \\
\hline $\mathrm{c} 5$ & 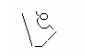 & c18 & Lat & c31 & $\overline{2 !}$ \\
\hline c6 & $\sigma$ & c19 & $e_{k j}$ & c32 & $\bar{q}$ \\
\hline c7 & $\bar{k}$ & c20 & 2 & c33 & $V_{6}$ \\
\hline c8 & $1-$ & c21 & $x$ & c34 & $\begin{array}{l}\mid 0) \\
1-6 \\
\text { or }\end{array}$ \\
\hline c9 & 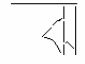 & c22 & $2 p$ & c35 & 2 \\
\hline $\mathrm{c} 10$ & $l_{\sim \gamma}^{a}$ & c23 & $\langle i$ & c36 & 乡 \\
\hline c11 & $\frac{4}{19}$ & c24 & $\sqrt{\xi}$ & c37 & $1^{\circ}$ \\
\hline c12 & $\overrightarrow{\angle 1}$ & c25 & 3 & c38 & $\begin{array}{l}\circ \\
\circ\end{array}$ \\
\hline c13 & $l_{-}$ & c26 & $2 !$ & c39 & $1 \%$ \\
\hline
\end{tabular}


Table 4.2: Truth Table for Bangla Alphabetic Character using 26-segments display

\begin{tabular}{|c|c|c|c|c|c|c|c|c|c|c|c|c|c|c|c|c|c|c|c|c|c|c|}
\hline & $2 y$ & $\mathrm{~F}$ & & & & & & & & & & & & & & & & & & & & \\
\hline & & & & & & & \begin{tabular}{l|l}
0 & 1
\end{tabular} & & 0 & & \begin{tabular}{l|l|l}
0 & 1
\end{tabular} & & & & & & & & & & & \\
\hline 1 & 7 & & 0 & & & & \begin{tabular}{l|l}
0 & 1
\end{tabular} & & & & \begin{tabular}{l|l}
0 & 1
\end{tabular} & & & & & & & & & & & \\
\hline & $\bar{y}$ & & 0 & \begin{tabular}{l|l}
0 & 1
\end{tabular} & & & \begin{tabular}{l|l}
0 & 0
\end{tabular} & & & & 01 & & & & & & & & & & & \\
\hline & & & 0 & \begin{tabular}{l|l}
0 & 1
\end{tabular} & 11 & & \begin{tabular}{l|l}
0 & 0
\end{tabular} & & & & \begin{tabular}{l|l}
0 & 1
\end{tabular} & & & & & & & & & & & \\
\hline & & 0 & 0 & 10 & 0 & 1 & \begin{tabular}{l|l}
0 & 1 \\
\end{tabular} & 1 & 0 & & \begin{tabular}{l|l}
0 & 0 \\
\end{tabular} & \begin{tabular}{|l|l}
0 & 1 \\
\end{tabular} & & & & & & & 0 & & \begin{tabular}{|l|l}
0 &
\end{tabular} & \\
\hline & & & 0 & 10 & 1 & & \begin{tabular}{l|l|l}
1 & 1
\end{tabular} & & 0 & 0 & \begin{tabular}{l|l}
0 & 0 \\
\end{tabular} & \begin{tabular}{|l|l|l|l|l|l|l|l|}
1 & 1
\end{tabular} & & $\begin{array}{lll}0 & 0 \\
\end{array}$ & 0 & 00 & & & 0 & & & \\
\hline & 8 & & 0 & 11 & 10 & 0 & \begin{tabular}{l|l}
0 & 0 \\
\end{tabular} & 0 & 0 & 0 & \begin{tabular}{l|l}
1 & 1 \\
\end{tabular} & 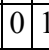 & 0 & \begin{tabular}{l|l}
0 & 1 \\
\end{tabular} & & $\begin{array}{lll}0 & 0 \\
\end{array}$ & & & & & & \\
\hline & 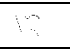 & & 0 & 11 & 11 & 0 & \begin{tabular}{l|l}
0 & 1
\end{tabular} & & & & \begin{tabular}{l|l}
0 & 1 \\
\end{tabular} & \begin{tabular}{|l|l|l|l|l|}
0 & 0 \\
\end{tabular} & & & & 而 & & & & & & \\
\hline & 4 & & 1 & & & & & & & & \begin{tabular}{l|l}
0 & 1 \\
\end{tabular} & \begin{tabular}{|l|l}
0 & 0 \\
\end{tabular} & & \begin{tabular}{|l|l|l|}
0 & 1 \\
\end{tabular} & & $\begin{array}{lll}0 & 1\end{array}$ & & & $\frac{10}{8}$ & & & \\
\hline & & & 1 & $0 \mid 0$ & & & & & 0 & & \begin{tabular}{l|l}
0 & 1
\end{tabular} & \begin{tabular}{|l|l|l|l|l|l|l|l|l|l|}
1 & 1 \\
\end{tabular} & & $\begin{array}{lll}0 & 0 \\
\end{array}$ & 0 & & 0 & & 0 & & & \\
\hline & & & 1 & \begin{tabular}{l|l}
0 & 1
\end{tabular} & & & 10 & & 0 & & \begin{tabular}{l|l}
0 & 1 \\
\end{tabular} & \begin{tabular}{|l|l}
1 & 1 \\
\end{tabular} & & \begin{tabular}{l|l}
0 & 0 \\
\end{tabular} & & 10 & 0 & & 10 & & & \\
\hline & & & 1 & \begin{tabular}{l|l|l} 
& 1
\end{tabular} & & ) & & & & 0 & 0.1 & & & & & & 0 & & & & & \\
\hline & & & 1 & & & 1 & \begin{tabular}{l|l}
0 & 0
\end{tabular} & & & 0 & \begin{tabular}{l|l}
0 & 0
\end{tabular} & & & & & 10 & & & & & & \\
\hline & & & 1 & & & & & & 0 & 0 & \begin{tabular}{l|l}
1 & 1 \\
\end{tabular} & \begin{tabular}{|l|l|l|}
0 & 1 \\
\end{tabular} & & \begin{tabular}{|l|l|l|}
0 & 1 \\
\end{tabular} & 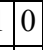 & $\begin{array}{l}00 \\
\end{array}$ & 1 & & 0 & & & \\
\hline & & & 1 & 11 & 10 & 0 & \begin{tabular}{l|l}
0 & 0 \\
\end{tabular} & 0 & 0 & 0 & \begin{tabular}{l|l}
0 & 0 \\
\end{tabular} & \begin{tabular}{|l|l}
1 & 0 \\
\end{tabular} & & \begin{tabular}{l|l}
0 & 0 \\
\end{tabular} & & \begin{tabular}{l|l}
0 & 1 \\
\end{tabular} & 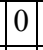 & & 0 & & & \\
\hline & & & 1 & 11 & 11 & 1 & \begin{tabular}{l|l}
0 & 0 \\
\end{tabular} & & & 10 & 1 & \begin{tabular}{l|l}
0 & 1 \\
\end{tabular} & 100 & 0 & & & & & 100 & & 100 & \\
\hline & & & 100 & 00 & 00 & 0 & \begin{tabular}{l|l}
0 & 1 \\
\end{tabular} & & & & \begin{tabular}{l|l}
1 & 1 \\
\end{tabular} & \begin{tabular}{|l|l}
0 & 1 \\
\end{tabular} & & $\begin{array}{lll}0 & 0 \\
\end{array}$ & 0 & $\begin{array}{lll}0 & 0 \\
\end{array}$ & & & 0 & & 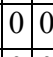 & \\
\hline & & & 10 & & 01 & & & & 1 & 0 & \begin{tabular}{l|l}
0 & 0
\end{tabular} & \begin{tabular}{|l|l}
0 & 1 \\
\end{tabular} & & \begin{tabular}{l|l}
0 & 0 \\
\end{tabular} & & 00 & 0 & & 0 & & \begin{tabular}{|l|l|l}
0 & 0 \\
\end{tabular} & \\
\hline & & & \begin{tabular}{ll|l} 
& 0 \\
\end{tabular} & $\begin{array}{llll}0 & 1\end{array}$ & $\begin{array}{l}1 \\
0\end{array}$ & 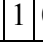 & \begin{tabular}{l|l}
0 & 0
\end{tabular} & & 1 & 0 & \begin{tabular}{l|l|l}
0 & 0
\end{tabular} & \begin{tabular}{|l|l|l|l|l|l|l|}
0 & 1
\end{tabular} & 11 & \begin{tabular}{|l|l|l|l|l|l|l|}
0 & 1
\end{tabular} & 10 & $\begin{array}{llll}0 & 0 \\
\end{array}$ & 0 & & 0 & & \begin{tabular}{l|l|l|l|l|l}
0 & 0
\end{tabular} & \\
\hline & & & 10 & $\begin{array}{ll}0 & 1\end{array}$ & 11 & it & \begin{tabular}{l|l}
0 & 1 \\
\end{tabular} & & 0 & 0 & \begin{tabular}{l|ll}
0 & 0 \\
\end{tabular} & \begin{tabular}{|l|l|}
1 & 1 \\
\end{tabular} & 11 & 00 & & 0 & U & & & & \begin{tabular}{|l|l|l}
0 & 0 \\
\end{tabular} & \\
\hline & & & 100 & 10 & & 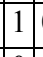 & \begin{tabular}{l|l}
0 & 0 \\
\end{tabular} & & & & & \begin{tabular}{|l|l|}
0 & 1 \\
\end{tabular} & & 0 & & 10 & & & 1 & & \begin{tabular}{|l|l}
1 & 0 \\
\end{tabular} & \\
\hline & & & 10 & & & & & 0 & U & & \begin{tabular}{l|l}
0 & 1 \\
\end{tabular} & \begin{tabular}{l|l}
0 & 0 \\
\end{tabular} & & \begin{tabular}{|l|l|} 
\\
\end{tabular} & & \begin{tabular}{l|l}
0 & 1 \\
\end{tabular} & & & 0 & & & \\
\hline & & & 100 & 11 & 100 & 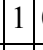 & \begin{tabular}{l|l}
0 & 0 \\
\end{tabular} & 1 & 1 & 0 & $\begin{array}{lll}0 & 0 \\
\end{array}$ & \begin{tabular}{|l|l}
0 & 1 \\
\end{tabular} & 10 & \begin{tabular}{l|l}
0 & 0 \\
\end{tabular} & & \begin{tabular}{l|l}
0 & 0 \\
\end{tabular} & 0 & & 0 & & \begin{tabular}{|l|l|l|}
0 & 1 \\
\end{tabular} & \\
\hline & & & 16 & & 11 & & & & & & \begin{tabular}{l|l}
0 \\
\end{tabular} & \begin{tabular}{l|l}
0 & 1 \\
\end{tabular} & 10 & \begin{tabular}{|l|l}
0 & 1 \\
\end{tabular} & & $\overline{10}$ & 1 & & 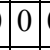 & & \begin{tabular}{|l|l}
0 & 1 \\
\end{tabular} & \\
\hline & & & & & & & & & & & 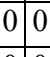 & \begin{tabular}{|l|l|l|}
0 & 1 \\
\end{tabular} & & 0 & & $\begin{array}{llll}0 & 0\end{array}$ & & & & & & \\
\hline 25 & & & & & & & & & & 0 & \begin{tabular}{l|l|}
0 \\
\end{tabular} & \begin{tabular}{l|l}
0 & 1 \\
\end{tabular} & & 0 & & & & & & & & \\
\hline & & & & & & & & & & & & $\begin{array}{llll}0 & 0 \\
\end{array}$ & 0 & $\begin{array}{lll}0 & 0 \\
\end{array}$ & J & $\begin{array}{lll}0 & 1\end{array}$ & 0 & & 0 & & \begin{tabular}{l|l|l|l|l}
0 & 0 \\
\end{tabular} & \\
\hline & & & . & \begin{tabular}{l|l}
0 & 1
\end{tabular} & & 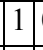 & 0 & 1 & & & 0 & \begin{tabular}{|l|l|l|}
1 & 1 \\
\end{tabular} & 10 & $\begin{array}{lll}0 & 0 \\
\end{array}$ & ) & 00 & 0 & & 0 & & \begin{tabular}{|l|l|l|l}
0 & 0 \\
\end{tabular} & \\
\hline & & & & & & & & & & & & & & 0 & & & & & & & \begin{tabular}{|l|l}
0 & 0 \\
\end{tabular} & \\
\hline 19 & & & 11 & & & & \begin{tabular}{l|l}
0 & 0 \\
\end{tabular} & & & \begin{tabular}{|l|l|l|l} 
& \\
\end{tabular} & \begin{tabular}{l|l}
0 & 0 \\
\end{tabular} & \begin{tabular}{|l|l|}
0 & 1 \\
\end{tabular} & $\mid 0$ & 0 & & $\begin{array}{lll}0 & 0 \\
\end{array}$ & & & 0 & \begin{tabular}{l|l}
0 & 0 \\
\end{tabular} & \begin{tabular}{l|l}
0 & 0 \\
\end{tabular} & \\
\hline & & & & & & & & & & & & \begin{tabular}{|l|l|l|}
0 & 1 \\
\end{tabular} & & \begin{tabular}{l|l}
0 & 1 \\
\end{tabular} & & 10 & & & & & \begin{tabular}{|l|l}
0 & 0 \\
\end{tabular} & \\
\hline & & & & & & 1 & 0 ( & & & & 0 & \begin{tabular}{|l|l}
0 & 0 \\
\end{tabular} & 0 & 1 & & $\begin{array}{lll}00 \\
\end{array}$ & & 11 & 0 & & \begin{tabular}{|l|l}
0 & 0 \\
\end{tabular} & \\
\hline & & & & & & & & & & & & 10 & 0 & 0 & & $\overline{11}$ & & & & \begin{tabular}{l|l}
0 & 0 \\
\end{tabular} & \begin{tabular}{l|l}
0 & 0 \\
\end{tabular} & \\
\hline & & & & & & & & & & & & 11 & 10 & & & & & & & & & \\
\hline & & & & & & & & & & & & & & & & & & & & & & \\
\hline & & & & & & & & & & & & & & & & & & & & & & \\
\hline
\end{tabular}




\begin{tabular}{|c|c|c|c|c|c|c|c|c|c|c|c|c|c|c|c|c|c|c|c|c|c|c|c|c|c|c|c|c|}
\hline 36 & & 1 & 0 & & 1 & $\begin{array}{l}0 \\
0\end{array}$ & 1 & 0 & 0 & 0 & 0 & & & 0 & & & 0 & & & & & & & & \begin{tabular}{l|l}
0 & 0
\end{tabular} & & & \\
\hline 37 & & & 0 & 0 & 10 & \begin{tabular}{l|l|l}
0 & 1
\end{tabular} & 1 & 0 & 0 & 0 & 0 & & & 0 & 1 & 1 & 0 & 0 & 10 & 0 & & & & & \begin{tabular}{l|l|l}
0 & 0
\end{tabular} & 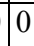 & 0 & \\
\hline 38 & & & 0 & 0 & 1 . & 10 & 1 & 0 & 0 & 0 & 0 & & & 0 & 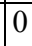 & 1 & 0 & 1 & 10 & 1 & & & & & \begin{tabular}{l|l}
0 & 0
\end{tabular} & 0 & & \\
\hline 39 & & & 0 & 0 & 1 & \begin{tabular}{l|l|l}
1 & 1
\end{tabular} & 1 & 0 & 0 & 0 & 0 & & & 0 & 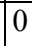 & 0 & 0 & \begin{tabular}{l|l|l}
1 & 1
\end{tabular} & 10 & 0 & & & & 0 & \begin{tabular}{l|l|l}
1 & 0
\end{tabular} & 0 & & \\
\hline 40 & & & 0 & 1 & 0 & $0 \mid c$ & 0 & 0 & 0 & $0 \mid$ & 0 & & & 1 & & 0 & 0 & $\begin{array}{lll}0 & 0\end{array}$ & & 0 & 1 & 0 & 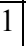 & & & & & \\
\hline 4 & & & 0 & 1 & 0 & \begin{tabular}{l|l}
0 & 1
\end{tabular} & 1 & 0 & 0 & 0 & 0 & & & 0 & 1 & 1 & 0 & $\begin{array}{lll}0 & 1\end{array}$ & $\begin{array}{lll}1 & 1\end{array}$ & 0 & V & 1 & 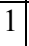 & & & & & 0 \\
\hline 42 & & & 0 & 1 & 0 & 10 & 1 & 0 & 0 & 0 & 0 & & & 0 & 1 & 1 & 0 & $\begin{array}{lll}0 & 1\end{array}$ & & 0 & o & 1 & 1 & & & & & \begin{tabular}{l|l}
0 & 0
\end{tabular} \\
\hline 43 & & & 0 & 1 & 0 & $1 \mid$ & 1 & 0 & 0 & 0 & 0 & & & 1 & 1 & 1 & 0 & $\begin{array}{lll}0 & 1\end{array}$ & & 0 & V & U & & U & & 0 & & 00 \\
\hline 44 & & 1 & 0 & 1 & $1 \mid$ & 0 & 1 & 1 & 0 & 1 & 0 & & & 0 & 0 & 1 & 1 & 10 & & 0 & V & U & & 0 & 0 & 0 & & \begin{tabular}{l|l}
0 & 0
\end{tabular} \\
\hline 45 & & 1 & 0 & 1 & 1 & 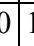 & 1 & 0 & 0 & 1 & 1 & & 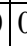 & 0 & 0 & 1 & 0 & 10 & & 0 & U & U & & 0 & \begin{tabular}{l|l}
0 & 0
\end{tabular} & ) & & \begin{tabular}{l|l}
0 & 0
\end{tabular} \\
\hline 46 & & 1 & 0 & 1 & & $1 \mid c$ & 1 & 0 & 0 & $0 \mid$ & 0 & & 0 & 0 & 1 & 1 & 0 & 11 & & 0 & 0 & | & & & \begin{tabular}{l|l}
0 & 0
\end{tabular} & & & $0 \mid 0$ \\
\hline 47 & & 1 & 0 & 1 & & $1 \mid$ & & 0 & 0 & $0 \mid$ & 0 & & & 1 & & 1 & 0 & & & 0 & & & & & & & & \begin{tabular}{l|l|l}
0 & 0
\end{tabular} \\
\hline 48 & & 1 & 1 & 0 & & 0 & & 0 & 1 & $0 \mid$ & 0 & & & & & 0 & 0 & & & & & & & & & & & 00 \\
\hline 49 & & 1 & & 0 & 0 & $\begin{array}{lll}0 & 1\end{array}$ & & & 0 & 0 & 0 & & & & & & 0 & & & & & & & & & & & $0 \mid 0$ \\
\hline & & 1 & & 0 & 0 & 10 & & 0 & 1 & 0 & 0 & & & & & 1 & 0 & \begin{tabular}{l|l}
0 & 0
\end{tabular} & & & & & & & & & & 0 \\
\hline
\end{tabular}

\section{Boolean Function for Bangla Alphabetic Characters}

From the Truth Table 1.2, we can write the following logic functions for different 26-segments in sum-of-product form:

$$
\begin{aligned}
& \mathrm{a}=\sum(0,1,2,3,4,5,12,15,17,18,19,20,22,23,24,25,27,29,31,33,34,35, \\
& \mathrm{b}=\sum(5,9,10,24,35,44) ; \\
& \mathrm{c}=\sum(0,1,4,5,7,8,16,19,21,27,48,50) ; \\
& \mathrm{d}=\sum(4,5,17,18,19,22,24,25,29,44,45) ; \\
& \mathrm{e}=\sum(17,18,22,25,29,45) ; \\
& \mathrm{f}=\sum(0,1,3,4,5,7,8,9,10,16,17,18,19,21,22,24,25,27,29,35,42,44,45,47,50) ; \\
& \mathrm{g}=\sum(16,40) ; \\
& \mathrm{h}=\sum(6,13,15,16,30,35,36,47) ; \\
& \mathrm{i}=\sum(0,1,2,3,6,7,8,9,10,11,13,16,21,26,27,28,30,35,40,43,47,48,49,50) ; \\
& \mathrm{j}=\sum(0,1,2,3,9,10,11,14,19,27,28,32,33,37,41,42,43,46) ; \\
& \mathrm{k}=\sum(0,1,2,3,4,5,6,9,10,12,13,15,16,17,18,19,20,22,23,24,25,27,28, \\
& \\
& =29,30,33,34,35,36,37,38,41,42,43,44,45,46,47,50) ; \\
& \mathrm{l}=\sum(4,5,9,10,17,18,19,24,44) ; \\
& \mathrm{m}=\sum(31,36,38,39,44,45,46,49) ;
\end{aligned}
$$




$$
\begin{aligned}
& \mathrm{n}=\sum(0,1,2,3,6,7,8,12,13,15,18,20,21,23,28,30,31,33,34,36,37,38,39,41,42,43,46) ; \\
& \mathrm{o}=\sum(19,41) ; \\
& \mathrm{p}=\sum(3,10,11,12,20,23,30,32,34,38,50) \\
& \mathrm{q}=\sum(7,8,14,21,26,32,40) \\
& \mathrm{r}=\sum(0,1,6,7,8,12,13,15,20,21,23,28,29,30,31,33,34,36,37,38,39,41,42,46) \\
& \mathrm{s}=\sum(0,1,6,7,8,12,13,14,15,20,21,23,26,28,30,31,32,33,34,36,37,38,40,41,42,46) ; \\
& \mathrm{t}=\sum(0,1,6,8,10,11,12,13,14,15,20,26,28,30,31,32,34,36,37,38,40,41,42,46) ; \\
& \mathrm{u}=\sum(6,20,39) \\
& \mathrm{v}=\sum(21) \\
& \mathrm{w}=\sum(12,21,33) \\
& \mathrm{x}=\sum(1,6,20,39) \\
& \mathrm{y}=\sum(2,3,4,5,8,10,11,22,23) \\
& \mathrm{z}=\sum(40)
\end{aligned}
$$

By using K-map or simulation software the above Boolean functions will be minimized and obtained the minimized logic circuits for Bangla Alphabetic Characters.

\section{A Comparative Study on Different Segmented Display Units}

Different segmented display units are compared based on display quality, number of characters to be displayed, number of segments, and average active segments. Display quality is considered as main criterion because it plays vital role for acceptability of the display unit in society. Maximum numbers of characters to be displayed, number of segments and average active segments per character are influenced by the cost and effectiveness of the display unit. In order to calculate the average active segments for a display model, divide total number of segments for the entire set of characters by total number of characters. For display quality, the entire set of characters of a display model compared with the standard Bangla fonts such as SutonnyMJ, RuposhreeMJ, DhanshirhirhiMJ, and RinkiyMJ. 17-segment and 14segment display units only provide the vowels of Bangla Alphabets, so the character sets of those models only compared with vowels of standard Bangla fonts. The appearances of some characters are near to the actual shape and some other stay below the marks [shown in 
Figure 1(b) and Figure 2(b)]. Thus the display quality is marked as fair. The entire set of characters for the display unit 31-segment model is not good at all, so it needs to remark as poor [shown in Figure 3(b)]. 44-segment display unit is only for Bangla consonants. Most of the characters are good enough with slightly moderate the basic shape of the original Bangla alphabets. Finally, our proposed 26-segments display unit covered the entire set of Bangla Alphabet characters with providing the original shape of the Bangla Characters. The comparison on different segmented display units for Bangla Characters is shown in Table 5.1 at a glance.

Table 5.1 Comparison on Different Segmented Display Units

\begin{tabular}{|c|c|c|c|c|c|}
\hline Criteria & $\begin{array}{c}17- \\
\text { Segment } \\
{[10]}\end{array}$ & $\begin{array}{c}14- \\
\text { Segment } \\
{[11]}\end{array}$ & $\begin{array}{c}31- \\
\text { Segment } \\
{[12]}\end{array}$ & $\begin{array}{c}\text { 44-Segment } \\
{[13]}\end{array}$ & $\begin{array}{l}\text { 26-Segment } \\
\text { [Proposed] }\end{array}$ \\
\hline $\begin{array}{l}\text { Number of } \\
\text { Segments }\end{array}$ & 17 & 14 & 31 & 44 & 26 \\
\hline $\begin{array}{l}\text { Number of } \\
\text { Characters to } \\
\text { be displayed }\end{array}$ & $\begin{array}{c}\text { Vowels } \\
\text { only } \\
11\end{array}$ & $\begin{array}{c}\text { Vowels } \\
\text { only } \\
11\end{array}$ & 36 & $\begin{array}{c}\text { Only } \\
\text { Consonants } \\
39\end{array}$ & $\begin{array}{c}\text { Entire set of } \\
\text { Alphabet } \\
51\end{array}$ \\
\hline $\begin{array}{l}\text { Average } \\
\text { Active } \\
\text { Segments }\end{array}$ & 6.5454 & 5.2727 & 7.055 & 12.641 & $\begin{array}{c}\text { Average for } \\
\text { Vowels } \\
7.916 \\
\text { Average for } \\
\text { Consonants } \\
6.35589 \\
\text { Finally } \\
\text { average for } \\
\text { entire set of } \\
\text { alphabets } \\
6.72549\end{array}$ \\
\hline $\begin{array}{l}\text { Display } \\
\text { Quality }\end{array}$ & Fair & Fair & Poor & $\begin{array}{l}\text { Good, but } \\
\text { shapes are } \\
\text { not same to } \\
\text { the original } \\
\text { characters }\end{array}$ & $\begin{array}{l}\text { Good with } \\
\text { maintaining } \\
\text { actual shape }\end{array}$ \\
\hline
\end{tabular}




\section{Conclusion}

In this paper, it has been shown how to activate different segment to have a successful display of all of the Bangla Alphabetic characters through our proposed 26-segment display system. Considering all factors, it is suggested that 26-segment display model is superior, in all respect, to four other models. To the best of our knowledge, this is the first proposed display system for Bangla Alphabetic characters both of vowels and consonants. From now on the proposed design may be frequently used rather than dot matrices to represent Bangla alphabetic characters which is more efficient, consumes less storage and more cost effective.

\section{References}

1. DOUGLAS V. HALL; Microprocessor and Interfacing, PP.4,54,164-165,227, 267.

2. WILKINSON, BARRZ \& HORROCKS, DAVID; Computer Peripherals, PP.67, 29-36.

3. RAHMAN, M.O., AZIM, M.A., CHOWDHURY, M.S., \& ISLAM, M.N.; (2003); Different Segment displays for Bangla, English and Arabic digits, 6th ICCIT 2003, Jahangirnagar University, PP. 299-302, December 19-21.

4. .......... (2002); Designing 11-Segment Display for Bangla Digits, 5th ICCIT 2002, East West University, Dhaka, PP. 237, December 27-28.

5. MAHMUD, N. \& KHAN, M.M.R., Designing 9-segment Display for Bangla Digits, Proceeding of the ICEECE 2003, Dhaka, Bangladesh, PP. 42-45, December 22-24.

6. MASUM, S.M., KHAN, M.S.I., RAHMAN, S.M.M., \& ALI, M. (2003); Designing 10-segment Display for Bangla Digits, Proceeding of the ICEECE 2003, Dhaka, Bangladesh, PP. 161-164, December 22-24.

7. ARIFIN, S.M.N., MEHEDY, L., \& KAYKOBAD, M. (2003); Segmented Display for Bangla Numerals: Simplicity vs. Accuracy, 6th ICCIT 2003, Jahangirnagar University, PP. 199-125, December 19-21.

8. HOSSAIN, G. \& HABIB, A.H.M.A. (2003), Desiging Numeric Characters Twin Display By-7 Segments, 6th ICCIT 2003, Jahangirnagar University, PP. 317-320, December 19-21.

9. TALUKDER, A.K.M.K.A. \& ROY, K. (2003); Development of 16-segment Multilingual Display Driver, Proceeding of the ICEECE 2003, Dhaka, Bangladesh, PP. 27-31, December 22-24.

10. ISLAM, A.K.M.N., MAHMUD, S.M.M., SHAHRIER, N., \& SATTAR, M.A. (2003); Designing 17-segment Display for Bangla Vowels, 6th ICCIT 2003, Jahangirnagar University, PP. 283-286, December 19-21. 
11. MASUM, S.M. \& AL-MAMUN, M.A. (2005); Designing 14-Segment Display for Bengali Vowels, Asian Journal of Information Technology, Grace Publications Network, Vol. 4 No. 2, PP. 178-184.

12. MAHMUD, S., AREFIN, A.S., \& KHAN, M.I. (2005); A New Approach for Displaying All Bengali Characters, Asian Journal of Information Technology, Grace Publications Network, Vol. 4 No. 2, PP.194-197.

13. MASUM, S.M., HAQUE, S.M., DASH, S.C., \& KABIR, K.F. (2005); Segmented Display System for Bengali Consonants, Asian Journal of Information Technology, Grace Publications Network, Vol. 4 No. 2, PP. 291-300, 2005.

14. SCHILDT, H.; The art of $C$, PP. 64-65. 